\title{
Rapid estimation of echocardiographic peak velocity of circumferential fibre shortening (peak Vcf) ${ }^{\star}$
}

\author{
PATRICK KO, DEREK R BOUGHNER, $\dagger$ WALTER J PAULSEN, $\dagger$ JOAN A PERSAUD
}

From the Cardiac Investigation Unit, Department of Medicine, University Hospital, and the

Department of Biophysics, University of Western Ontario, London, Ontario, Canada

SUMMARY A transparent grid for estimating peak Vcf directly from the echocardiograph recording is described. Estimates so obtained were compared with peak Vcf measurements made by an echocardiographic digitising technique and with the angiographic ejection fraction in 13 patients with normal left ventricular function and 17 patients with impaired left ventricular function. No patient had angiographic segmental wall motion abnormalities. Significant linear correlations were found between peak Vcf derived by the simple manual technique and peak Vcf determined by the digitising technique and also between peak Vcf derived by the simple manual technique and the angiographic ejection fraction. Peak Vcf derived by the simple manual technique was found to be a reliable predictor of ventricular function, being more than 1.4 circumferences/s in 11 of 13 patients with angiographic ejection fractions $\geq 55 \%$ and less than 1.4 in 14 of 17 patients with angiographic ejection fractions $<55 \%$ (sensitivity $82 \%$, specificity $85 \%$ ). Thus, peak Vcf is an echocardiographic index that can be estimated rapidly and accurately without the need for digitising equipment and is well suited to general clinical use.

The echocardiographic peak velocity of circumferential fibre shortening (peak Vcf) has been proposed by several investigators as a reliable index of left ventricular performance, ${ }^{1-7}$ and recent reports suggest that it may be a more sensitive measure of left ventricular function than other currently used echocardiographic indices. ${ }^{36}$ The major limitation of peak Vcf, however, is that its measurement requires an $\mathrm{X}-\mathrm{Y}$ digitiser and a programmable computer, devices that are expensive and not readily available to many echocardiographers. The general clinical use of peak Vcf has therefore been limited. It is the purpose of this report to present a simplified and inexpensive method of estimating the echocardiographic peak Vcf and to validate its accuracy by comparing the peak Vcf so measured with the peak Vcf determined from the echocardiogram by a digitising technique and also with the left ventricular ejection fraction determined angiographically.

\section{Methods}

Echocardiographic studies were performed on 30

« Supported by a grant from the Ontario Heart Foundation.

+Drs Boughner and Paulsen are Ontario Heart Foundation Research Fellows.

Received for publication 19 December 1980 patients undergoing diagnostic cardiac catheterisation. None of the patients had arteriographic evidence of significant ( $>50 \%$ stenosis) coronary artery disease or segmental wall motion abnormalities. On the basis of their angiographic ejection fraction (EF), the patients were divided into two groups. One group consisted of 13 patients with normal left ventricular function (EF $>55 \%)$. This group included seven patients with chest pain syndrome but no evidence of organic heart disease and six patients with minimal valvular heart disease. The second group comprised 17 patients who had evidence of impaired left ventricular function $(\mathrm{EF}<55 \%)$ and included four patients with congestive cardiomyopathy and 13 patients with valvular heart disease.

\section{ECHOCARDIOGRAPHY}

Echocardiograms were obtained using a Unirad, Series C echocardiographic unit equipped with a Honeywell 1858 strip chart recorder. Patients were studied in the slight left lateral position with the transducer positioned in the fourth or fifth left intercostal space. The left ventricular minor axis diameter was recorded by directing the ultrasound beam just inferior to the mitral valve, and recordings were made at a paper speed of 100 $\mathrm{mm} / \mathrm{s}$ (Fig. 1). 


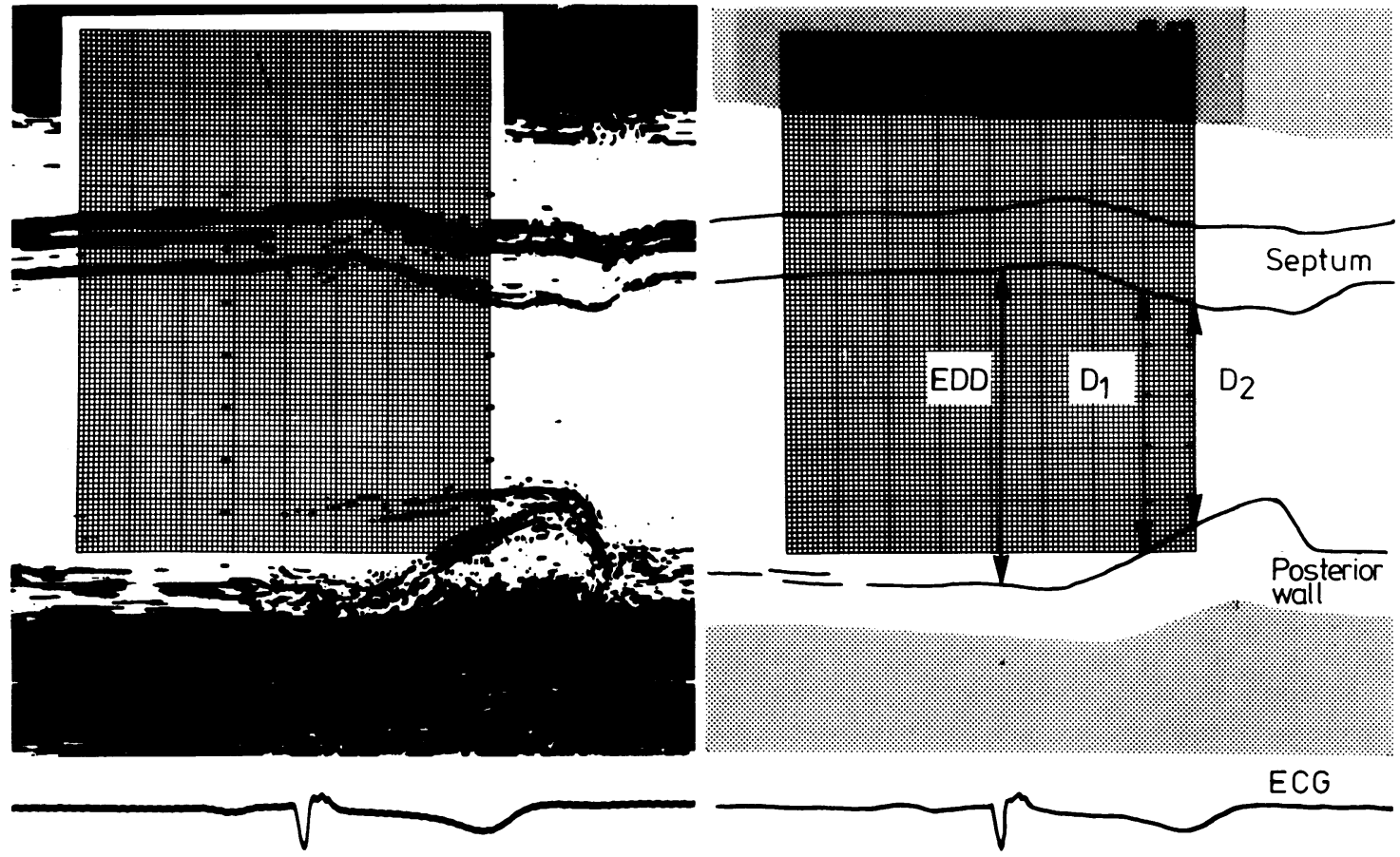

Fig. 1 Left:Left ventricularminor axisechogram recorded at $100 \mathrm{~mm} / \mathrm{spaper} \mathrm{speed.} \mathrm{The} \mathrm{transparent} \mathrm{grid} \mathrm{has} \mathrm{been} \mathrm{placed} \mathrm{directly} \mathrm{onto}$ the recording for the manual calculation of the diameter change during mid-systole. Right: Schematic representation of the measurements. Two ventricular diameter measurements, $D_{1}$ and $D_{2}$, are made $100 \mathrm{~ms}$ apart during mid-systole. Their difference is the diameter change per $100 \mathrm{~ms}$ and when multiplied by 10 gives diameter change/s. This value is then divided by end-diastolic diameter $(E D D)$ measured by aligning the grid with the ' $R$ ' wave of the electrocardiogram. Thus peak $V c f=\frac{\left(D_{1}-D_{2}\right) \times 10}{E D D} \mathrm{circ} / \mathrm{s}$

\section{CALCULATION OF PEAK VCF BY DIGITISING TECHNIQUE}

Analysis of the instantaneous left ventricular dimension and calculation of its peak rate of change was performed using a Hewlett-Packard Model 1838 desk top computer, a Model 986A digitiser, and X-Y plotter combination by a method modified from that of Upton and Gibson. ${ }^{57}$ The recordings of the left ventricular echograms were placed on a digitising table and the endocardial echoes of the septum and posterior wall were digitised beginning at the onset of the ' $R$ ' wave and ending with the ' $T$ ' wave. The computer noted the position of these two echoes and automatically derived the instantaneous left ventricular diameter. It normalised this diameter measurement by dividing by the initial diameter and this ratio was then displayed by the $\mathrm{X}-\mathrm{Y}$ plotter as a "displacement ratio" versus time curve (Fig. 2a). Peak Vcf in circ/s was obtained manually by calculating the slope of the tangent to the mid portion of this curve.

We elected to make this last calculation manually rather than to use the computer generated first derivative of the displacement ratio: time curve (Fig. $2 \mathrm{~b})$ as has been described by Gibson and Brown. ${ }^{2}$ We encountered difficulties in the reproducibility of the latter because of the sensitivity of our computer program to minor irregularities in the initial curve. This noise was generated by "hand shake" when the operator was tracing the septal and posterior wall echoes on the digitiser board. This produced erroneously high peaks of velocity on many of the first differential recordings. Such irregularities were easily compensated for by the tangent method giving good reproducibility. ${ }^{7}$ For each patient, peak Vcf was obtained in three beats by this technique and the average was used to provide the final digitised peak Vcf estimate.

\section{CALCULATION OF PEAK VCF BY SIMPLE} MANUAL TECHNIQUE

On examination of a typical computer print-out of the left ventricular minor axis diameter displacement ratio versus time curve (Fig. 2a), we see that as the minor axis diameter decreases the slope of the curve is steepest during the first two-thirds of systole and that during this portion of systole, the curve approximates a straight line for about $140 \mathrm{~ms}$. Thus, the rate of diameter change reaches a maximum (or peak) soon after ejection begins, maintains a maximum rate of change during 

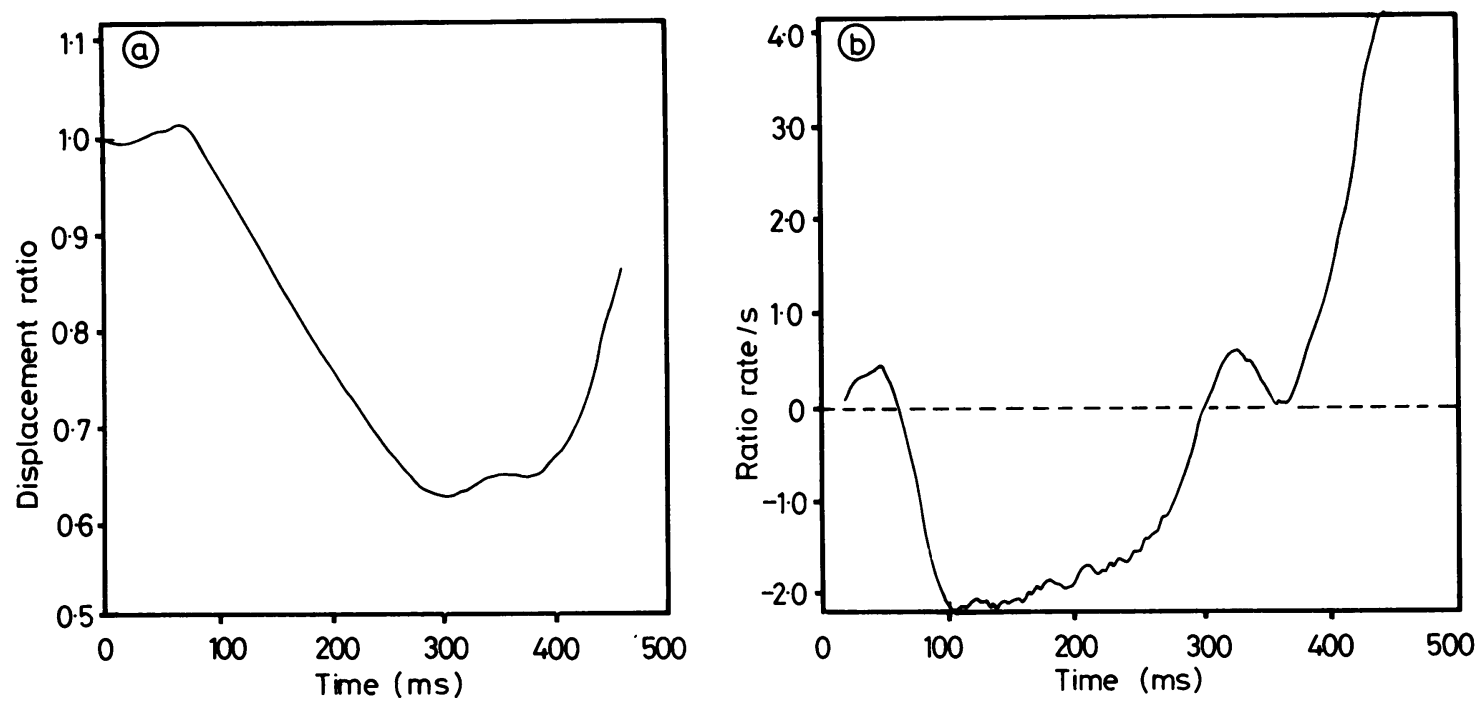

Fig. 2 (a) Computer-digitiser plot of displacement ratio versus time. The displacement ratio is the computer calculated instantaneous left ventricular diameter divided by the initial end-diastolic diameter $(E D D)$. The graph begins at the ' $R$ ' wave and ends at the ' $T$ ' wave and shows the change in ventricular diameter with time during systole. (b) Computer calculated time course of Vcf from $2 a$. The first derivative of the displacement ratio versus time plot (that is, instantaneous Vcf) is shown to remain relatively constant from $100-250$ ms during mid-systole.

mid-ejection, and falls off later in systole as cavity size diminishes.

This is confirmed by analysing the corresponding computer print-out of the time curve of Vcf (Fig. 2b), which shows that with the onset of ejection, Vcf increases rapidly to its peak, maintains a velocity close to that peak during early systolic ejection, and then falls off during late systole. In addition, visual inspection of a left ventricular echogram (Fig. 1) shows that the maximum rate of rise of the posterior wall endocardium and the maximum rate of descent of the septal endocardium occur during early systolic ejection. Therefore, it is not necessary to measure Vcf throughout the whole of systole to obtain an estimate of peak Vcf. The peak rate of change of diameter can be established easily and rapidly by measuring the left ventricular minor axis diameter during the first two-thirds of systole at two points $100 \mathrm{~ms}$ apart. Dividing this peak rate of diameter change by the end-diastolic diameter then provides the normalised peak Vcf in circ/s.

In the present study, this simplified technique of estimating peak $\mathrm{Vcf}$ was performed in the following manner. By placing a transparent $8 \times 10 \mathrm{~cm}$ grid directly on the left ventricular echocardiogram, the left ventricular diameter was measured during early systole at two points $10 \mathrm{~mm}$ apart (Fig. 1). At a paper speed of $100 \mathrm{~mm} / \mathrm{s}$ this $10 \mathrm{~mm}$ interval represented $100 \mathrm{~ms}$. Thus, the difference between the two measurements represented the change of ventricular diameter in that $100 \mathrm{~ms}$ of systole when the diameter was changing most rapidly. When multiplied by 10 this value provided the simplified peak rate of change of diameter per second.

Dividing this value by the end-diastolic diameter (again measured using the grid) provided the normalised peak $\mathrm{Vcf}$ in circ/s. Since all measurements were performed using the transparent grid, it was not necessary to correct for magnification on the echocardiogram. Thus, peak $V c f=\frac{\left(D_{1}-D_{2}\right) \times 10}{E D D} \mathrm{circ} / \mathrm{s}$ (Fig. 1), where $D_{1}$ and $\mathrm{D}_{2}$ represent sequential measurements of left ventricular minor axis diameter 100 ms apart during midsystole and EDD represents end-diastolic diameter. The peak Vcf for each patient was taken as the average of peak Vcf values derived from three beats. When estimating the peak rate of diameter change from the echocardiograms, particular care was taken to obtain measurements at a time during early to mid-systole when the velocity of motion of both the septum and posterior wall appeared to be at their maximum.

\section{RE PRODUCIBILITY}

Measurements of four successive beats in eight randomly selected patients were carried out by two observers independently in order to establish the beat to beat and interobserver variation in the peak Vcf calculations by both the digitised technique and the simple manual technique. One way analysis of variance indicated good reproducibility as evidenced by a coefficient of variation of less than $5 \%$ for the digitised technique and less than $8 \%$ for the simple manual technique. Four patients had repeat echocardiograms performed one week after their initial study and peak Vcf derived by both methods did not differ significantly 
between the two studies. Thus, reproducibility did not seem to be a problem.

\section{CARDIAC CATHETERISATION}

All patients underwent clinically indicated cardiac catheterisation within 72 hours of their echocardiographic assessment. Left ventricular cineangiography was performed in the $30^{\circ}$ right anterior oblique and $60^{\circ}$ left anterior oblique projections. Left ventricular ejection fractions were calculated from the right anterior oblique cineangiograms using the single plane area-length technique. ${ }^{8}$ Coronary arteriography was performed in all patients older than 40 years.

\section{STATISTICAL ANALYSIS}

The left ventricular echocardiograms were analysed by two experienced echocardiographers who did not have

Table Clinical, echocardiographic, and angiographic data

\begin{tabular}{lll}
\hline & Peak $V c f(c i r c / s)$ & \\
\cline { 3 - 4 } Diagnosis $\quad$ Age/Sex $\quad$ Manual Digitised & $\begin{array}{l}\text { Angio- } \\
\text { graphic EF } \\
(\%)\end{array}$
\end{tabular}

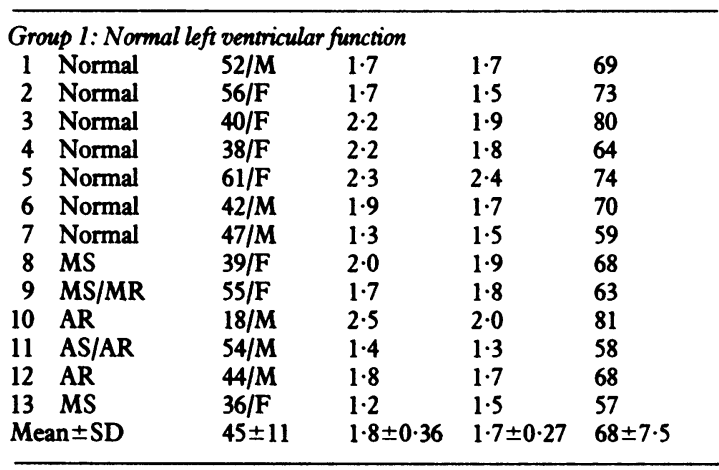

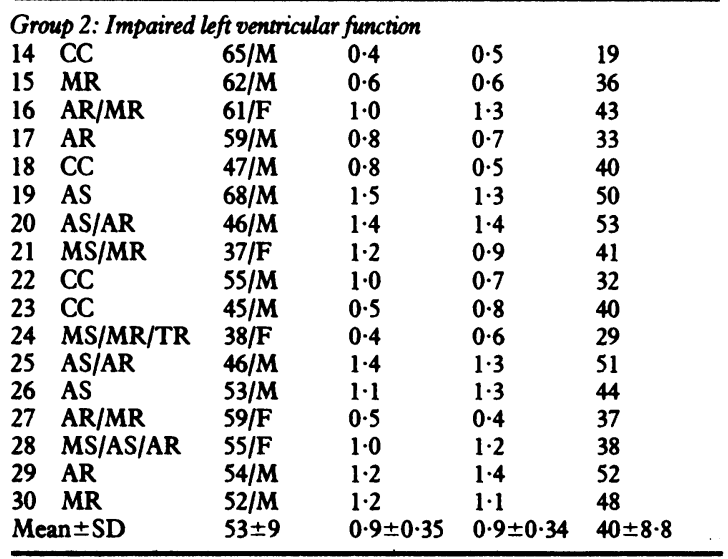

MS, mitral stenosis; MR, mitral regurgitation; AS, aortic stenosis; AR, aortic regurgitation; CC, congestive cardiomyopathy. prior knowledge of the patients' clinical diagnosis or results of their angiographic ejection fractions. Each echocardiographer was assigned one or the other of the two methods for deriving peak Vcf and calculated peak Vcf for each patient using the method assigned to him, but without knowledge of the results obtained by the alternative method. Statistical analysis was carried out using one way analysis of variance and Student's $t$ test. Regression analysis was carried out using two way variable analysis.

\section{Results}

The individual data of the 30 patients are summarised in the Table. An angiographic ejection fraction $>55 \%$ was considered to represent normal ventricular function whereas an ejection fraction $<55 \%$ was considered to be depressed. Normal echocardiographic peak Vcf values for our laboratory have been established previously in 20 subjects and range from 1.4 to $2.2 \mathrm{circ} / \mathrm{s}$ with a mean of $1 \cdot 8 \pm 0.21 \mathrm{circ} / \mathrm{s}$.

Peak Vcf derived by the simple manual technique was significantly higher in patients with normal left ventricular function $(1.8 \pm 0.36 \mathrm{circ} / \mathrm{s})$ than in patients with depressed left ventricular function $(0.9 \pm 0.35$ circ/s; $\mathbf{p}<0.001$, Table). Despite the statistically significant difference between the two groups some overlap of values for peak Vcf was noted. Peak Vcf was "normal" ( $\geq 1.4 \mathrm{circ} / \mathrm{s})$ in 11 of 13 patients with normal left ventricular function (angiographic ejection fraction $\geq 55 \%$ ) and depressed in 14 of 17 patients with a depressed angiographic ejection fraction (sensitivity $82 \%$, specificity $85 \%$ ).

Peak Vcf determined by the digitising technique was $1.7 \pm 0.27 \mathrm{circ} / \mathrm{s}$ in patients with normal left ventricular function and $0.9 \pm 0.34 \mathrm{circ} / \mathrm{s}$ in patients with impaired left ventricular function. These values were not significantly different from those obtained by the simple manual technique. With the digitising technique, however, there was slightly less overlap between values for normal and abnormal left ventricular function. Thus, peak Vcf was normal in 12 of 13 patients with normal left ventricular function and depressed in 15 of 17 patients with impaired left ventricular function (sensitivity $88 \%$, specificity $92 \%$ ).

Peak Vcf derived by the simple manual technique was found to correlate well with both peak Vcf derived by the digitising technique $(r=0.93, p<0.001$, Fig. 3$)$ and with the angiographic ejection fraction $(r=0.92$, p $<0.001$, Fig. 4).

\section{Discussion}

In recent years, it has been shown that echocardiographic peak Vcf is a reliable non-invasive index of left ventricular performance ${ }^{1-7}$ and some authors have 


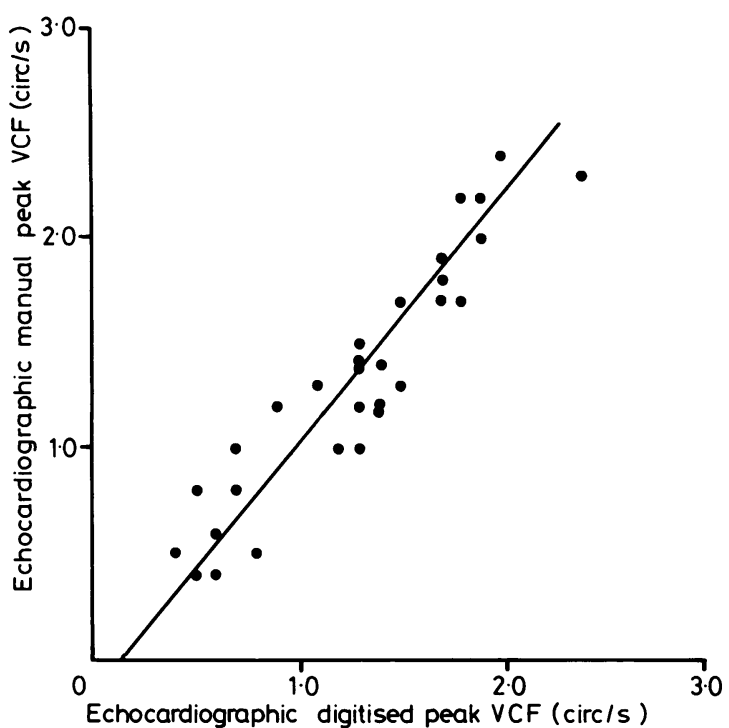

Fig. 3 The linear correlation between the estimate of peak $V c f$ obtained using the grid overlay and the estimate of peak $V c f$ obtained from the computer-digitiser plat of the ventricular diameter is shown $(r=0.93, p<0.001)$.

recommended it as superior to the other echocardiographic indices currently in use. ${ }^{36}$ This recommendation is not based on an increased sensitivity of peak Vcf to the ventricular inotropic state but simply the fact that the variable contains fewer approximations than are found in the other indices. For example, the lower sensitivity and specificity exhibited by mean Vcf have been attributed to both the inaccuracies in estimating ventricular ejection time without a carotid pulse

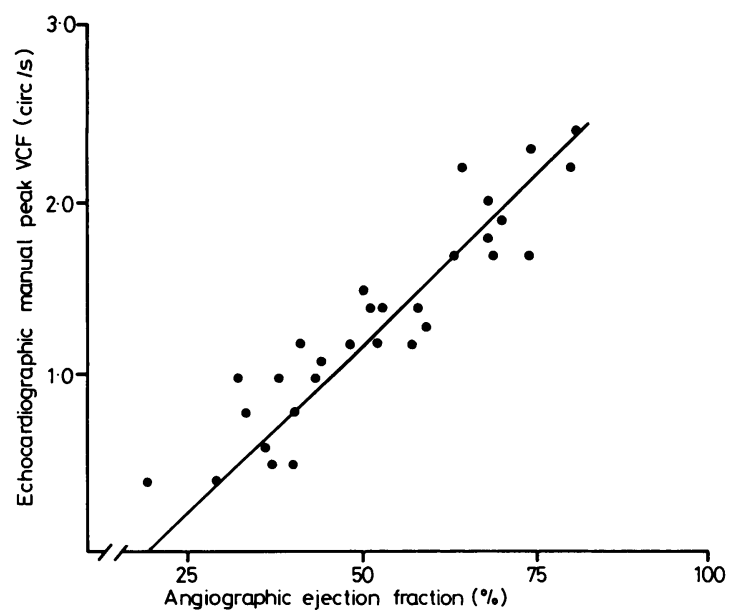

Fig. 4 The correlation between the rapid peak Vcf estimate and the angiographic ejection fraction is shown $(r=0.92, p<0.001)$. tracing and to the difficulties in determining the exact point of minimum ventricular minor axis diameter. ${ }^{6}$ Both these estimates are integral parts of the mean Vcf formula and similar problems exist with the echocardiographic estimates of ejection fraction ${ }^{9}$ and percentage fractional shortening. ${ }^{5}$

In spite of the improved accuracy offered by peak Vcf over other indices, the clinical application of peak Vcf to the routine assessment of left ventricular performance has been limited by the requirement for either laborious manual techniques ${ }^{14}$ or more expensive digitising techniques that can be time-consuming to perform..$^{23-7}$ This problem can be overcome by using the transparent grid technique described herein which allows a simple and rapid estimate of peak $\mathrm{Vcf}$ by estimating the slope of the left ventricular dimension curve and correlates well with peak Vcf determined by a more elaborate digitising technique.

We selected the calculation of angiographic ejection fraction as our validation technique for the usefulness of the simple manual peak Vcf measurements. Though there is no unequivocal basis on which an absolute measure of myocardial function can be defined, this angiographic measurement is the single most widely used index of left ventricular function in clinical practice. ${ }^{10} \mathrm{~A}$ good linear correlation was obtained for the two measurements and we therefore concluded that the simple manual peak Vcf technique was a satisfactory means of separating patients with normal and abnormal left ventricular function. As already indicated, there was some overlap in peak Vcf values between the two groups. On closer examination, it was apparent that this overlap occurred only in patients with borderline angiographic ejection fractions (50 to $59 \%$ ), and separation between patients with unequivocally normal or depressed ejection fractions was excellent.

The simplified technique for measuring peak Vcf that we have presented relies on establishing the rate of change of left ventricular minor axis diameter for a 100 ms period during early systolic ejection. This approach could reduce the reliability of the peak Vcf estimate if the $100 \mathrm{~ms}$ portion of systole selected for measurement does not actually contain the peak rate of diameter change. Previous studies analysing the time course of the velocity of diameter reduction during systole, however, indicate that the peak velocity is invariably achieved during early ejection in both normal and diseased hearts. ${ }^{11-13}$ Precise identification of this portion of ejection when the velocities of both the septum and posterior wall are clearly at their maximum is facilitated by recording left ventricular echograms at a rapid paper speed (preferably $100 \mathrm{~mm} / \mathrm{s}$ ).

In conclusion, this report has presented a simple, inexpensive manual technique for estimating peak Vcf echocardiographically. The method offers a practical 
alternative to the more elaborate digitising technique previously described.

The authors wish to thank Dr Brian Finlay for writing the computer programs and Mr Steve Mesjarik of the University Hospital Instructional Resources Department for producing the transparent grids.

\section{References}

1 Paraskos JA, Grossman W, Saltz S, Dalen JF, Dexter L. A noninvasive technique for the determination of velocity of circumferential fibre shortening in man. Circ Res 1971; 29: $610-5$.

2 Gibson DG, Brown D. Measurement of instantaneous left ventricular dimension and filling rate in man, using echocardiography. Br Heart f 1973; 35: 1141-9.

3 Quinones MA, Gaasch WH, Cole JS, Alexander JK. Echocardiographic determination of left ventricular stress-velocity relations in man: with reference to the effects of loading and contractility. Circulation 1975; 51: 689-700.

4 Boughner DR, Nolan J, Rechnitzer P. Usefulness of echocardiographic peak velocity of circumferential fibre shortening. In: White $\mathrm{D}$, Barnes $\mathrm{R}$, eds. Ultrasound in medicine, vol. 2. New York: Plenum Press, 1976: 21-30.

5 Upton MT, Gibson DG. The study of left ventricular function from digitised echocardiograms. Prog Cardiovasc Dis 1978; 20: 359-84.

6 Wilson JR, Reichek N. Echocardiographic indices of left ventricular function: a comparison. Chest 1979; 76: 441-7.

7 Paulsen WJ, Boughner DR, Friesen A, Persaud JA. Ventricular response to isometric and isotonic exercise: echocardiographic assessment. Br Heart f 1979; 42: 521-7.

8 Sandler H, Dodge HT. The use of single plane angiocardiograms for the calculation of left ventricular volume in man. Am Heart f 1968; 75: 325-34.

9 Segal BL, Kotler MN. Echocardiography in current perspective. Cardiovasc Clin 1978; 9: 343-55.
10 Gibson DG. Clinical assessment of left ventricular function. In: Hamer J, ed. Recent advances in cardiology, vol. 7. New York: Churchill-Livingstone, 1977: 315-47.

11 Gault JH, Ross J Jr, Braunwald E. Contractile state of the left ventricle in man: instantaneous tension-velocitylength relations in patients with and without disease of the left ventricular myocardium. Circ Res 1968; 22: 451-61.

12 Peterson KL, Uther JB, Shabetai R, Braunwald E. Assessment of left ventricular performance in man: instantaneous tension-velocity-length relations obtained with the aid of an electromagnetic velocity catheter in the ascending aorta. Circulation 1973; 47: 924-35.

13 Mathey DG, Decoodt PR, Allen HN, Swan HJC Abnormal left ventricular contraction pattern in systolic click-late systolic murmur syndrome. Circulation 1977; 56: 311-5.

\section{Appendix}

The transparent grids used in this study were produced in the following manner. A sheet of $K \& E 10 \times 10$ graph paper was first copied using a large format process camera and a flat field process lens. The image on the ground glass was reduced such that each square measured one millimetre. The film used for reproduction was a high contrast line material, Kodalith Ortho 6556 type 3 in $4^{\prime \prime} \times 5^{\prime \prime}$ size. This film was processed using the appropriate high contrast chemistry and the resulting negative was used to make as many contact prints as desired. These contact prints or positives were produced on 4 " $\times 5^{\prime \prime}$ sheets of the same high contrast line film and processing resulted in the transparent film grids used for the measurements.

Requests for reprints to Dr Derek R Boughner, Cardiac Investigation Unit, University Hospital, PO Box 5339, Terminal A, London, Ontario, Canada N6A 5A5. 
ERRATA : to be pasted page 28. Vol. 5 (January, 1943)

Page 28-

Line 8. For " (30)" read " (20)".

Line 11. For "Fig. 2B" read " Fig. 3B".

Page 29. Legend to Fig. 3, line 6. For " a 1 " read " an 11 ".

Page 30. Line 12. For " true " read "large".

Page 31-

Line 7. For " is " read "was".

Line 15. For " and "read" or".

\section{REFERENCES}

18. Ann. intern. Med. (In press.)

21. (1942). Amer. Heart J., 24, 627.

39. (1942) J. Lab. clin. Med., 28, 303.

\section{ADDENDUM}

While this article was in press, Schwartz and Marcus (Schwartz, S. P., and Marcus, H. (1942) Amer. Rev. Tuberc., 46, 35) reported a number of cases of our Type IV and several of our Type VI pattern. These patterns occurred predominantly in cases of chronic cor pulmonale. From their autopsy data in 7 such cases, as well as in 2 additional cases of congenital heart disease, the patterns appear to be most commonly associated with predominant hypertrophy of the right ventricle. However, they also occurred in the absence of ventricular hypertrophy when the right ventricle was dilated. 